\section{Fluorescent microplate-based analysis of protein-DNA interactions I: immobilized protein}

\author{
Zhan-Ren Zhang ${ }^{1}$ David Palfrey ${ }^{1}$, David A. Nage ${ }^{1}$, Peter A. Lambert ${ }^{1}$, \\ Robert A. Jessop ${ }^{2}$, Albert F. Santos ${ }^{2}$, and Anna V. Hine ${ }^{1}$ \\ ${ }^{1}$ Aston University, Birmingham and ${ }^{2}$ Amersham Biosciences, Amersham plc, \\ Cardiff, UK
}

BioTechniques 35:980-986 (November 2003)

A simple protein-DNA interaction analysis has been developed using a high-affinity/highspecificity zinc finger protein. In essence, purified protein samples are immobilized directly onto the surface of microplate wells, and fluorescently labeled DNA is added in solution. After incubation and washing, bound DNA is detected in a standard microplate reader. The minimum sensitivity of the assay is approximately $0.2 \mathrm{nM}$ DNA. Since the detection of bound DNA is noninvasive and the protein-DNA interaction is not disrupted during detection, iterative readings may be taken from the same well, after successive alterations in interaction conditions, if required. In this respect, the assay may therefore be considered real time and permits appropriate interaction conditions to be determined quantitatively. The assay format is ideally suited to investigate the interactions of purified unlabeled DNA binding proteins in a high-throughput format.

\section{INTRODUCTION}

Historically, protein-DNA interactions are studied by gel shift analysis [electrophoretic mobility shift assay (EMSA)] (1). The methodology is inexpensive but effectively "freezes" a protein-DNA interaction in time and cannot generate real-time data with respect to binding conditions because the interaction is detected only after the protein-DNA complex has been electrophoresed. More recently, surface plasmon resonance (SPR) (2) has been employed to monitor protein-DNA interactions. This technique has the advantage that it generates real-time data (both for kinetic and other analyses) but is expensive. Fluorescence correlation spectroscopy and fluorescence anisotropy are also used to study proteinDNA interactions (3), but none of these techniques are readily adaptable to a high-throughput format. Alternatively, protein-DNA interactions (of bacteriophage displaying individual zinc finger proteins) may be quantified by ELISA (4), which is both inexpensive and amenable to high-throughput (e.g., by the generation of protein-DNA interaction arrays) (5). High-throughput analysis can also be achieved by using modified nitrocellulose filter binding assays (6), but again, these techniques cannot generate data in real time.

Current advances in both EMSA and SPR have also begun to address throughput. For example, EMSA has been adapted to a capillary electrophoresis format (7) and, by using a fluorescently labeled DNA sequence, detection of the retarded complex has been automated using a DNA sequencer (8). Similarly, by linking SPR to charge-coupled device (CCD) imaging, arrays with immobilized DNA to detect protein-DNA interactions have been developed (9), and SPR detectors for the analysis of 2-D arrays are under commercial development (10). Thus, SPR may soon address both throughput and real-time analysis, but the process is likely to be costly.

We wished to combine the advantages of all three approaches to generate an assay that is inexpensive and requires no specialized instrumentation in a format that is readily amenable to high-throughput screening but that also allows one of the principle advantages of real-time study: the iterative analysis of samples after successive changes in interaction conditions. Our assay is based on direct protein immobilization in microplate wells, with fluorescently labeled DNA added free in solution.

\section{MATERIALS AND METHODS}

\section{Materials}

Oligonucleotides for gene synthesis (DAN1-6) and binding site oligonucleotides $\mathrm{BSH}$ and $\mathrm{BSHc}$ were obtained from Applied Biosystems (Warrington, UK) and MWG-Biotech (Milton Keynes, UK), respectively. Table 1 lists their sequences. Plasmid pGEX2TK was obtained from Amersham Biosciences (Little Chalfont, UK). Enzymes for DNA manipulation were obtained from either MBI Fermentas (St. Leon-Rot, Germany) or New England Biolabs (Beverly, MA, USA). Shallow well black fluoroimmunoassay (FIA) medium binding microplates and standard black FIA high binding microplates were obtained from Greiner Bio-One (Stonehouse, UK). NUNC Black MaxiSorp ${ }^{\mathrm{TM}}$ microplates were obtained from Fisher Scientific (Loughborough, UK).

\section{Zinc Finger Gene Construction and Expression}

The following pairs of oligonucleotides, DAN1/DAN4 (fragment Z1), DAN2/DAN5 (fragment Z2), and DAN3/DAN6 (fragment Z3), were annealed, prepared for cloning, and the fragments were inserted individually into SmaI-digested dephosphorylated pUC19 (MBI Fermentas) as previously described (11). After sequence verification, the inserts were excised by combined AgeI/EcoRI digestion (employing the EcoRI site from the pUC19 polylinker); XmaI/XbaI digestion (employing the $X b a I$ site of the pUC19 polylinker); and XmaI/EcoRI digestion, respectively; and gel-purified in $1 \%$ SeaPlaque ${ }^{\circledR}$ agarose (Flowgen, Ashby de la Zouch, UK). Fragments $\mathrm{Z} 1$ and $\mathrm{Z} 2$ were ligated, and the product was digested with $A g e$ I, treated with phosphatase, and then purified on silica beads. The combined Z1/Z2 product was ligated with fragment $Z 3$, and the completed construct was then ligated into EcoRI-digested pUC19. After sequence verification, the zinc finger gene was excised by combined Bam HI/EcoRI digestion and ligated into similarly digested pGEX2TK to generate plasmid pGST-ZFH. 
Table 1. Oligonucleotide Sequences for Gene Construction (DAN1-6) and Protein-DNA Interaction Analysis

\begin{tabular}{|c|c|}
\hline Oligonucleotide & Sequence \\
\hline DAN1 & $\begin{array}{l}\text { 5'-GGATCCGAGAAACTTCGTAATGGTTCGGGCGACCCAGGAAAGAAGAAACAGCATGCGT- } \\
\text { GCCCAGAGTGTGGTAAGAGCTTCAGTCAATCC-3' }\end{array}$ \\
\hline DAN2 & $\begin{array}{l}\text { 5'-CCCGGGGAGAAACCGTACAAGTGTCCAGAATGCGGGAAGTCCTTTAGTCGCAGCGAC- } \\
\text { GAATTACAACGTCATCAGCGCACTCACACCGGT-3' }\end{array}$ \\
\hline DAN3 & $\begin{array}{l}\text { 5'-CCCGGGGAAAAGCCATATAAATGCCCTGAATGTGGCAAGTCTTTCAGCCGTAGTGATCATCT- } \\
\text { GTCTCGCCATCAACGCACGCATCAGAAC-3' }\end{array}$ \\
\hline DAN4 & $\begin{array}{l}\text { 5'-ACCGGTATGTGTACGTTGGTGGCGCTGCAGATCAGAGGATTGACTGAAGCTCTTACCA- } \\
\text { CACTCTGGGCACGCATGCTGTTTCTTCTTTCC-3' }\end{array}$ \\
\hline DAN5 & $\begin{array}{l}\text { 5'-ACCGGTGTGAGTGCGCTGATGACGTTGTAATTCGTCGCTGCGACTAAAGGACTTCCCG- } \\
\text { CATTCTGGACACTTGTACGGTTTCTCCCCGGG-3' }\end{array}$ \\
\hline DAN6 & $\begin{array}{l}\text { 5'-GAATTCTCATTTCTTGTTCTGATGCGTGCGTTGATGGCGAGACAGATGATCACTACGGCT- } \\
\text { GAAAGACTTGCCACATTCAGGGCATTTATA-3' }\end{array}$ \\
\hline $\mathrm{BSH}$ & $5^{\prime}-$ Су3-T 10 GGGGCGGCTT $10^{-3}$ \\
\hline $\mathrm{BSHc}$ & $5^{\prime}-$ Су3-A ${ }_{10} A_{G C C G C C C C A} 0^{-3^{\prime}}$ \\
\hline
\end{tabular}

Escherichia coli BL21 cells (EMD Biosciences, Madison, WI, USA) were transformed freshly with pGST-ZFH, and a single colony was inoculated into $200 \mathrm{~mL} \mathrm{LB}$ medium containing $50 \mu \mathrm{g} / \mathrm{mL}$ ampicillin and $1 \%$ glucose, which was then incubated with shaking at $37^{\circ} \mathrm{C}$ overnight. The resulting culture was added to $1800 \mathrm{~mL}$ of the same medium prewarmed to $37^{\circ} \mathrm{C}$ and further incubated at $37^{\circ} \mathrm{C}$ until an $\mathrm{A}_{600}$ of approximately 0.4 was reached. The cells were then harvested by centrifugation at 2,500× $g$ for $7 \mathrm{~min}$ and resuspended in $2 \mathrm{~L}$ of $\mathrm{LB}$ medium containing 50 $\mu \mathrm{g} / \mathrm{mL}$ ampicillin (no glucose) that had been prewarmed to $30^{\circ} \mathrm{C}$. Isopropyl$\beta$-D-thiogalactoside (IPTG) was added to a final concentration of $0.2 \mathrm{mM}$, and the cells were incubated with shaking at $30^{\circ} \mathrm{C}$ overnight. The cells were again harvested by centrifugation at $2,500 \times g$ for $7 \mathrm{~min}$, and the glutathione-Stransferase (GST) fusion protein was purified as previously described (11). Protein concentration was estimated by using the Bio-Rad Protein Assay kit (Bio-Rad Laboratories, Hercules, CA,
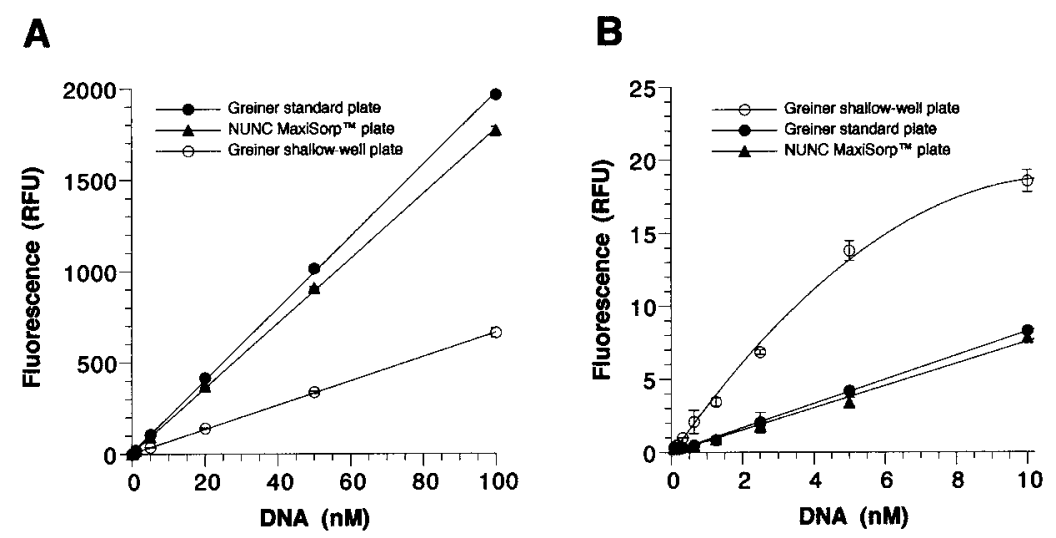

Figure 1. Sensitivity of both free and bound DNA detection in three types of black microplates. (A) Calibration curves for Cy3-labeled double-stranded binding site DNA (concentrations as indicated) were measured, and data were fitted by linear regression (in each case, $R>0.99$ ). (B) GST-ZFH protein (133 $\mathrm{nM} ; 4$ pmol shallow-well plates and 47 pmol standard well plates) was immobilized, and protein-DNA interaction analyses (DNA concentrations as indicated) were performed as described. The error bars represent standard deviation (note that some error bars do not exceed the area occupied by the data markers). RFU, relative fluorescence units.

USA) according to the manufacturer's instructions, using bovine serum albu$\min$ (BSA) as a standard.

\section{Double-Stranded DNA for Use in Protein-DNA Interaction Analyses}

Binding site oligonucleotides BSH and BSHc $(100 \mu \mathrm{M})$ were combined and heated to $95^{\circ} \mathrm{C}$ for 2 min and then cooled to $25^{\circ} \mathrm{C}$ at a speed of $1{ }^{\circ} \mathrm{C} / \mathrm{min}$ in a thermal cycler (PTC-200 ${ }^{\mathrm{TM}}$; MJ Research, Waltham, MA, USA). The DNA was aliquoted and stored in the dark at $4^{\circ} \mathrm{C}$ until required.

\section{Microplate-Based Detection of Protein-DNA Interactions}

Protein $(133 \mathrm{nM}$ in $20 \mathrm{mM}$ Tris$\mathrm{HCl}, \mathrm{pH} 8.5$ ) was loaded into the microplate wells [30 $\mu \mathrm{L}$ per shallow-well plate $(4 \mathrm{pmol})$ and $350 \mu \mathrm{L}$ per other plates $(47 \mathrm{pmol})]$, and the plate was incubated at $25^{\circ} \mathrm{C}$ for $1 \mathrm{~h}$ and subsequently at $37^{\circ} \mathrm{C}$ for $90 \mathrm{~min}$. Unbound protein was removed by the rapid inversion and subsequent washing of each well three times in $30 \mu \mathrm{L} / 350 \mu \mathrm{L}$ TBST [0.01 M Tris-HCl, pH 7.4, 0.9\% $\mathrm{NaCl}(\mathrm{w} / \mathrm{v}), 0.1 \%$ Tween $\left.{ }^{\circledR} 20(\mathrm{v} / \mathrm{v})\right]$. The plates were then blocked with similar volumes of tris-buffered saline (TBS) containing $2 \% \mathrm{BSA}(\mathrm{w} / \mathrm{v})$ for 30 min and washed three times in TBST as described above. 
Cy ${ }^{\mathrm{TM}} 3$-labeled double-stranded binding site DNA in TBS $(30 \mu \mathrm{L} / 350 \mu \mathrm{L})$ was then added in concentrations as indicated (Figures 1-3), and the plate was wrapped in aluminium foil and incubated at room temperature for $1 \mathrm{~h}$. Unless otherwise stated, the plates were then washed three times in TBST. After the removal of the final wash by inversion, fluorescence was measured in a SPECTRAmax ${ }^{\circledR}$ Gemini XS plate reader (Molecular Devices, Sunnyvale, CA, USA) with excitation and emission wavelengths of 510 and $565 \mathrm{~nm}$, respectively, and a cut-off filter of $550 \mathrm{~nm}$. All measurements (both calibration curves and protein-DNA interaction analyses) were performed in triplicate, and the data were fitted using KaleidaGraph ${ }^{\circledR}$ Version 3.08d (Synergy Software, Reading, PA, USA).

\section{RESULTS AND DISCUSSION}

A gene encoding the consensus sequence zinc finger protein previously described by Desjarlais et al. (12), which binds with high specificity and affinity to the DNA sequence 5'-GGGGCG-



Figure 2. Measurement of apparent dissociation constant $\left(K_{d a p p}\right)$ between glutathione-Stransferase (GST)-ZFH and its target DNA sequence. GST-ZFH (4 pmol) was immobilized in black shallow-well microplates, and proteinDNA interaction analyses were performed with 0-100 nM double-stranded binding site DNA. For the quantification of bound DNA, a calibration was measured over the same concentration range and the data fitted by linear regression $(R>0.99)$. $\mathrm{K}_{\mathrm{dapp}}$ was calculated by plotting bound versus free DNA with data fitted to the equation $\mathrm{y}=$ $\mathrm{m} 1 * \mathrm{~m} 0 /(\mathrm{m} 0+\mathrm{m} 2)$ where $\mathrm{y}=$ bound DNA; $\mathrm{m} 1=$ total available binding sites; $\mathrm{m} 0=$ free DNA; $\mathrm{m} 2$ $=\mathrm{K}_{\mathrm{d}}$ (dissociation constant) (17). Free DNA was calculated by subtracting bound from total DNA. The error bars represent standard deviation.
GCT-3', was synthesized employing $E$. coli-preferred codons (13) to maximize subsequent expression. The gene was then expressed as an $\mathrm{N}$-terminal fusion to GST, and the resulting protein, named GST-ZFH, was purified by affinity chromatography. GST-ZFH was used to develop a protein-DNA interaction assay that would be amenable to highthroughput screening.

Initially, three black microplates, the shallow well medium binding plate, the standard high binding plate, and the MaxiSorp plate, were evaluated with respect to sensitivity. All are 96-well format, but the small volume plate consists of 96 shallow slope-sided wells with maximum sample loading capacities of $30 \mu \mathrm{L}$, whereas the other two plates have standard cylindrical wells with a maximum loading volumes of $350 \mu \mathrm{L}$. All three plates may be accommodated in standard microplate readers. First, we determined that each plate gave a linear response to the Cy3-labeled DNA. In each case, a linear response was seen from 0.2-100 nM DNA (Figure 1A).

We then evaluated each plate for its utility in monitoring protein-DNA interactions. Preliminary experiments employing radiolabeled protein demonstrated that, in analogy to the immobilization of protein antigens in a standard ELISA, the zinc finger proteins could be immobilized directly onto the surface of microplate wells (data not shown).
GST-ZFH protein was therefore immobilized onto the surfaces of the wells in each microplate, and Cy3-labeled target DNA was added. After incubation and washing, immobilized DNA was quantified (Figure 1B). Although we anticipate that much higher quantities of protein will be immobilized in the large volume plates, we were interested to find that over similar ranges of DNA concentration, the greatest sensitivity was obtained using the small volume plate in which the well sides are sloped (Figure 1B). Interestingly, in this plate, DNA binding was starting to saturate at the highest concentrations used, while the other two plates were still in the initial linear phase of binding over this concentration range of DNA. Because it is beneficial in high-throughput screening to minimize reagent costs and volumes, the shallow-well plate was adopted for future use.

The fluorescence-based assay was then used to measure the apparent dissociation constant of GST-ZFH with its target DNA sequence (Figure 2). For reference, free QDR-RER-RHR protein (the ZFH component of GST-ZFH) is reported to have a dissociation constant $\left(\mathrm{K}_{\mathrm{d}}\right)$ of $2 \mathrm{nM}$ when measured by conventional gel shift analysis (12). In our assay, the analysis of the binding data generated an apparent dissociation constant $\left(\mathrm{K}_{\mathrm{dapp}}\right)$ value of $7.3 \mathrm{nM} \pm 1.0(n=$ $3 \pm$ SEM). The agreement between these
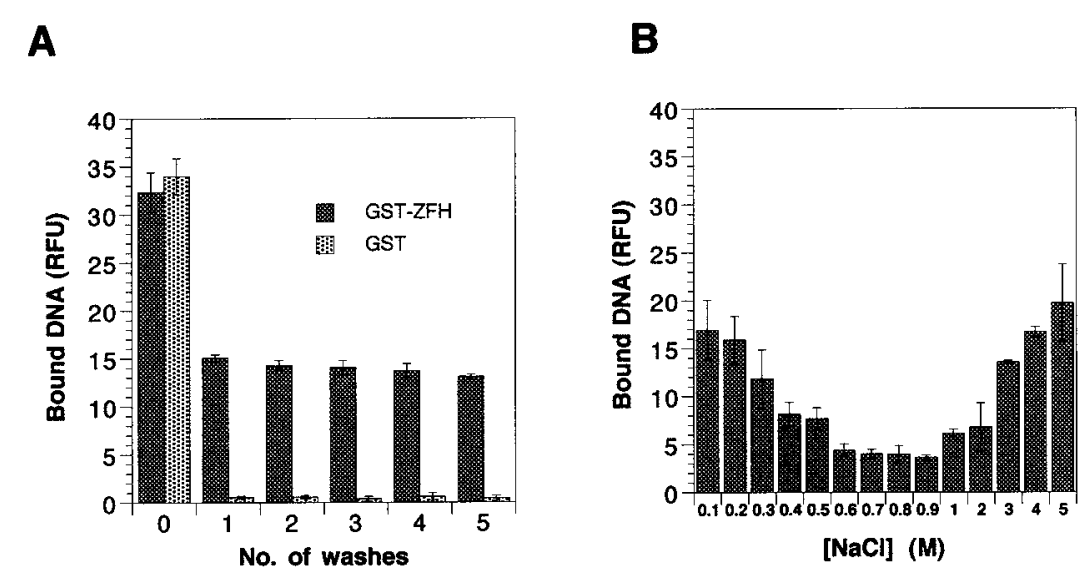

Figure 3. The effect of varying elution conditions on the glutathione-S-transferase (GST)-ZFH/DNA interaction. All analyses were performed using GST-ZFH or GST protein (4 pmol) as indicated, immobilized in black shallow-well microplates, and incubated with $10 \mathrm{nM}$ double-stranded binding site DNA. (A) The effect of multiple washings. After the addition of DNA, the plate was quantified, washed once in tris-buffered saline (TBS), and requantified. Washing and subsequent requantification were repeated four times. (B) The effect of differing $\mathrm{NaCl}$ concentrations. Unbound DNA was removed by washing once in TBS containing $\mathrm{NaCl}$ (concentrations as indicated). The error bars represent standard deviation. RFU, relative fluorescence units. 
values, although they were obtained by different methods, suggests that the GST domain has relatively little effect on DNA affinity.

Finally, we examined the effect of washing and buffer composition on the protein-DNA interaction. Ideally, washing should eliminate background interaction (noise) but leave the specific interaction (signal) intact. Normally, however, the number and stringency of washes are a matter of precedent and/or guesswork. By exploiting the iterative potential of our assay, we were able to identify both the ideal number of washes and the effect of varying salt concentration on the zinc finger-DNA interaction. We first immobilized both GST-ZFH and GST (to represent background signal) proteins. Standard fluorescence-based assays were performed, but instead of washing three times and then quantifying bound DNA, fluorescence was quantified as soon as the DNA had been added and after each subsequent washing step. Figure 3A demonstrates that the majority of unbound/weakly bound DNA is removed from GST after one wash, whereas DNA binding by GST-ZFH is largely unaffected by subsequent multiple washes. We therefore concluded that one wash is optimal to remove unbound DNA in this assay format.

We next investigated the effect of increasing stringency by evaluating the salt concentration that would be required to elute the GST-ZFH protein from the DNA. A fluorescence-based protein-DNA interaction assay was performed as above, but elutions were carried out by a single wash of buffer that contained varying concentrations of $\mathrm{NaCl}$. Figure 3B demonstrates that $0.6-0.9 \mathrm{M} \mathrm{NaCl}$ is the most effective in disrupting the protein-DNA interaction, while the interaction is neither disrupted by $0.1 \mathrm{M} \mathrm{NaCl}$ nor, interestingly, by 5 $\mathrm{M} \mathrm{NaCl}$. (We confirmed that the Cy3labeled DNA does not precipitate or adhere to the surface of the microplate in the presence of $5 \mathrm{M} \mathrm{NaCl}$.) Thus, these high and low salt conditions equate with washing in buffer alone (Figure 3A).

In conclusion, we have developed a simple microplate-based fluorescent assay that is ideally suited to the analysis of protein-DNA interactions of purified unlabeled proteins. In concept, our assay is similar to an inverted ELISA, except that no extraneous reagents are required for the detection of bound ligand. This noninvasive assay may be used to quantitatively optimize assay conditions. For example, we discovered that for the DNA binding protein GST-ZFH, one wash was sufficient to remove nonspecific DNA binding. Moreover, specific binding is relatively unaffected by both high (1-5 M) and low (0-0.3 M) $\mathrm{NaCl}$ concentrations but is disrupted effectively by $0.6-0.9$ $\mathrm{M} \mathrm{NaCl}$. We propose that in addition to the utility of a simple high-throughput assay format, the ability to determine ideal assay conditions easily should substantially enhance the quality of data obtained from such analyses. In principle, the format is also similar to an inverted biopanning experiment $(14,15)$, except that within biopanning, it is not possible to quantify the protein-DNA interaction prior to elution. In the following paper (16), we examined an inversion of our assay for use with crude mixtures of proteins, a method that directly mimics a quantitative biopanning experiment.

\section{ACKNOWLEDGMENTS}

This work was supported by BBSRC grant no. 92/B14245 (Z.-R.Z.) and BBSRC/Amersham Biosciences studentships (D.P. and D.A.N.). We thank Drs. D.R. Poyner and A.J. Sutherland (Aston University) for helpful discussions and Drs. M.D. Hughes and R.A.J. Darby (Aston University) for critical readings of the manuscript.

\section{REFERENCES}

1.Kerr, L.D. 1995. Electrophoretic mobility shift assay. Methods Enzymol. 254:619-632.

2.McDonnell, J.M. 2001. Surface plasmon resonance: towards an understanding of the mechanisms of biological molecular recognition. Curr. Op. Chem. Biol. 5:572-577.

3.Sevenich, F.W., J. Langowski, V. Weiss, and K. Rippe. 1998. DNA binding and oligomerization of NtrC studied by fluorescence anisotropy and fluorescence correlation spectroscopy. Nucleic Acids Res. 26:1373-1381.

4.Choo, Y. and A. Klug. 1994. Selection of DNA binding sites for zinc fingers using rationally randomized DNA reveals coded interactions. Proc. Natl. Acad. Sci. USA 91:11168-11172.

5.Bulyk, M.L., X.H. Huang, Y. Choo, and G.M. Church. 2001. Exploring the DNA- binding specificities of zinc fingers with DNA microarrays. Proc. Natl. Acad. Sci. USA 98: 7158-7163.

6.Wong, I. and T.M. Lohman. 1993. A doublefilter method for nitrocellulose-filter binding: application to protein-nucleic acid interactions Proc. Natl. Acad. Sci. USA 90:5428-5432.

7.Janini, G.M., R.J. Fisher, L.E. Henderson, and H.J. Issaq. 1995. Application of capillary zone electrophoresis for the analysis of proteins, protein-small molecules, and protein-DNA interactions. J. Liq. Chromatogr. 18: 3617-3628.

8.Ruscher, K., M. Reuter, D. Kupper, G. Trendelenburg, U. Dirnagl, and A. Meisel. 2000. A fluorescence based non-radioactive electrophoretic mobility shift assay. J. Biotechnol. 78: 163-170.

9.Brockman, J.M., A.G. Frutos, and R.M. Corn. 1999. A multistep chemical modification procedure to create DNA arrays on gold surfaces for the study of protein-DNA interactions with surface plasmon resonance imaging. J. Am. Chem. Soc. 121:8044-8051.

10.Baird, C.L. and D.G. Myszka. 2001. Current and emerging commercial optical biosensors. J. Mol. Rec. 14:261-268.

11.Woodgate, J., D. Palfrey, D.A. Nagel, A.V. Hine, and N.K.H. Slater. 2001. Protein-mediated isolation of plasmid DNA by a zinc finger-glutathione $S$-transferase affinity linker. Biotech. Bioeng. 79:411-418.

12.Desjarlais, J.R. and J.M. Berg. 1993. Use of a zinc-finger consensus sequence framework and specificity rules to design specific DNA binding proteins. Proc. Natl. Acad. Sci. USA 90:2256-2260

13.Nakamura, Y., T. Gojobori, and T. Ikemura. 2000. Codon usage tabulated from the international DNA sequence databases: status for the year 2000. Nucleic Acids Res. 28:292-292.

14.Rebar, E.J. and C.O. Pabo. 1994. Zinc finger phage: affinity selection of fingers with new DNA-binding specificities. Science 263: 671-673.

15.Segal, D.J., B. Dreier, R.R. Beerli, and C.F. Barbas III. 1999. Toward controlling gene expression at will: selection and design of zinc finger domains recognising each of the 5'-GNN-3' DNA target sequences. Proc. Natl. Acad. Sci. USA 96:2758-2763.

16.Zhang, Z.-R., M.D. Hughes, L.J. Morgan, A.F. Santos, and A.V. Hine. 2003. Fluorescent microplate-based analysis of protein-DNA interactions II: immobilized DNA. BioTechniques 35:988-996.

17.Choo, Y. and A. Klug. 1993. A role in DNA binding for the linker sequences of the first three zinc finger proteins of TFIIIA. Nucleic Acids Res. 21:3341-3346.

Received 12 June 2003; accepted 2 September 2003.

Address correspondence to Anna V. Hine, School of Life and Health Sciences, Aston University, Aston Triangle, Birmingham, B4 7ET, UK.e-mail:a.v.hine@aston.ac.uk 